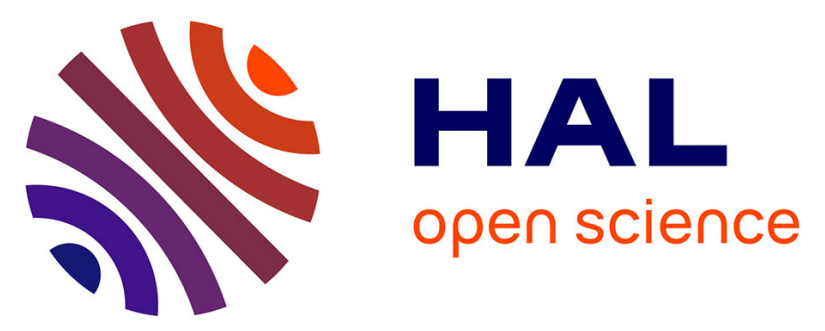

\title{
Vélocimétrie Doppler sous champ électrique: technique et application à l'étude de la mobilité électrophorétique des colloïdes et des polyélectrolytes
}

\author{
J.P. Dalbiez, K. Tabti, P.J. Derian, M. Drifford
}

\section{- To cite this version:}

J.P. Dalbiez, K. Tabti, P.J. Derian, M. Drifford. Vélocimétrie Doppler sous champ électrique: technique et application à l'étude de la mobilité électrophorétique des colloïdes et des polyélectrolytes. Revue de Physique Appliquée, 1987, 22 (9), pp.1013-1024. 10.1051/rphysap:019870022090101300 . jpa-00245637

\section{HAL Id: jpa-00245637 https://hal.science/jpa-00245637}

Submitted on 1 Jan 1987

HAL is a multi-disciplinary open access archive for the deposit and dissemination of scientific research documents, whether they are published or not. The documents may come from teaching and research institutions in France or abroad, or from public or private research centers.
L'archive ouverte pluridisciplinaire HAL, est destinée au dépôt et à la diffusion de documents scientifiques de niveau recherche, publiés ou non, émanant des établissements d'enseignement et de recherche français ou étrangers, des laboratoires publics ou privés. 


\title{
Vélocimétrie Doppler sous champ électrique : technique et application à l'étude de la mobilité électrophorétique des colloïdes et des polyélectrolytes
}

\author{
J. P. Dalbiez, K. Tabti $(*)$, P. J. Derian et M. Drifford (**) \\ CEA-IRDI-DESICP-Département de Physico-Chimie, \\ CEN, Saclay, 91191 Gif sur Yvette Cedex, France
}

(Reçu le 7 juillet 1986, révisé le 20 février 1987, accepté le 26 mai 1987)

\begin{abstract}
Résumé. - La diffusion de lä lumière sous champ électrique est une technique importante pour caractériser la charge et l'hydrodynamique des colloïdes, macromolécules, et polyélectrolytes. Nous avons construit un appareil pour la mesure de la mobilité électrophorétique en utilisant une technique différentielle Doppler sous champ électrique. Le système d'optique de base employé est dérivé de la vélocimétrie Doppler laser. Deux faisceaux parallèles, polarisés dans le même sens, sont focalisés dans une cellule d'électrophorèse. Le croisement des faisceaux produit des franges d'interférence. La modulation de l'intensité reçue par le détecteur est due à la variation lumineuse provoquée par les particules qui traversent les franges blanches et noires (cette explication, simple bien que non rigoureuse, permet néanmoins une description physique de l'expérience). La modulation du signal est analysée par un analyseur de fréquence et le déplacement du spectre de fréquence est directement relié à la vitesse électrophorétique des macromolécules. Le grand avantage de cette méthode est que la lumière peut être collectée par une large ouverture et ainsi toute l'intensité est utilisée pour le signal. Dans une première partie, nous introduisons le principe et la théorie de base de la vélocimétrie Doppler sous champ électrique (VDCE). Ensuite nous décrivons en détail l'appareillage et particulièrement la cellule électrophorétique ; celle-ci est rectangulaire en verre avec des électrodes de platine. Le champ électrique est ainsi bien défini et nous avons étudié le profil théorique des vitesses pour en déduire la position des points stationnaires. Le point d'interférence des faisceaux, placé au niveau des points stationnaires, permet de mesurer avec précision la vitesse et ainsi de minimiser les effets d'électro-osmose. La procédure expérimentale est décrite pour obtenir les valeurs de la mobilité électrophorétique (signe de la charge des polyions ou des colloïdes), le profil expérimental des vitesses dans la cellule, et la largeur du pic de l'intensité diffusée sous champ électrique. L'application de cette technique sur des collö̈des minéraux $\left(\mathrm{Al}(\mathrm{OH})_{3}, \mathrm{TiO}_{2}\right)$ pour la détermination des potentiels Zeta est montrée, ainsi que l'influence du $\mathrm{pH}$ sur le signe des charges. L'étude expérimentale de la mobilité électrophorétique de polyélectrolytes linéaires et flexibles est discutée et comparée à des modèles théoriques. L'évolution de la mobilité du polystyrène sulfonate de sodium (PSSNA) avec la concentration en solution aqueuse sans sel montre un «cross-over » à la transition critique de recouvrement $C^{*}$. En régime semi-dilué, la variation de la mobilité est en accord avec la théorie de Manning. La dépendance de la mobilité avec le sel ajouté est également en accord avec les évolutions théoriques. Enfin, le phénomène de condensation des contre-ions sur les poly-ions est mis en évidence par l'évolution de la mobilité électrophorétique en fonction de la concentration en polyélectrolyte à $\kappa$ constant ( $\kappa$ étant le paramètre d'écrantage de Debye).
\end{abstract}

Abstract. - Electrophoretic light scattering is an important technique for characterizing the charge and hydrodynamic behaviour of colloid, macromolecule and polyelectrolyte solutions. We have constructed an apparatus for the measurement of electrophoretic mobility in using a differential Doppler technique in an electrical field. The basic optical system is derived from laser Doppler velocimetry. Two parallel laser beams, polarized in the same direction, are focused into an electrophoretic cell. The cross-beam intersection produces some interference fringes. The "modulation" of the intensity at the detector is due to the variation of the illumination of particles as they are driven through the fringes (this simple representation of the phenomena is not really rigourous but gives good results). The modulation is detected by a spectrum analyser and the

(*) Stagiaire DGRST.

(**) Correspondant. 
Doppler frequency shift is correlated to the electrophoretic velocity of macromolecules. The high advantage of this method is that the light may be collected over a wide aperture, and the scattered light contributes usefully to the signal. In a first part, we have introduced the background theory of Doppler velocimetry under electric field (DVEF). Then, we describe in detail the apparatus and mainly the electrophoretic cell in glass, which has a rectangular shape in order to have a good definition of the electric field. We have studied the theoretical and experimental velocity profiles in the cell in order to deduce the stationary plan. With this technique (cross beam intersection), we can exactly measure the velocity on a stationary point and thus, minimize the electroosmosis phenomena. A critical experiment procedure is discussed to obtain, the value of the electrophoretic mobility (sign of the polyion charge), the experimental velocity profile into the cell and the width of scattered peak under electric field. The use of set up apparatus is described with some tests in the field of colloids. The application of this technique on mineral colloidal solutions $\left(\mathrm{Al}(\mathrm{OH})_{3}, \mathrm{TiO}_{2}\right)$ is described to obtain Zeta potentials and to show the influence of $\mathrm{pH}$ on the sign of charges. The experimental study of electrophoretic mobilities of linear and flexible polyelectrolytes is discussed and compared to theoretical approaches. The concentration dependence of mobilities in sodium polystyrene sulfonate solutions without added salt shows a cross-over at the critical concentration $C^{*}$. In semi-dilute regime, the experimental behaviour is in agreement with Manning's theory. The salt concentration dependence is also in agreement. The condensation phenomena on polyelectrolytes is shown by investigating the dependence of electrophoretic mobility as a function of polyelectrolyte concentration at constant $\kappa$ ( $\kappa$ is the Debye Huckel screening parameter).

\section{Introduction.}

La diffusion quasi élastique de la lumière sous champ électrique (DQLCE) est une technique originale pour étudier les propriétés dynamiques des solutions de polyélectrolytes. En effet, les phénomènes électrocinétiques de surface des polyions, les interactions entre petits ions (contre-ions) et polyions peuvent être analysés en mesurant la mobilité électrophorétique des polyélectrolytes en fonction des conditions du milieu (force ionique, concentration, $\mathrm{pH}$ etc...).

Le couplage entre l'électrophorèse et la diffusion de la lumière a été proposée en 1971 par B. Ware et W. Flygare [1] pour étudier la migration de macromolécules chargées sous l'effet d'un champ électrique. Un déplacement Doppler du spectre diffusé, proportionnel au champ électrique appliqué $(E)$ permet une mesure précise de la vitesse électrophorétique $(V)$ et de la constante de proportionnalité c'est-à-dire la mobilité électrophorétique $(U)$.

$$
V=U E \text {. }
$$

La valeur des mobilités électrophorétiques varie entre 6 et $2 \times 10^{-4} \mathrm{~cm}^{2} / \mathrm{s} / \mathrm{V}$ pour la plupart des macromolécules chargées.

L'application d'un champ électrique entre 10 et $50 \mathrm{~V} / \mathrm{cm}$ entraîne une vitesse de l'ordre de 40 à $200 \mu \mathrm{m} / \mathrm{s}$. Le déplacement Doppler, de la lumière diffusée par un laser He-Ne est alors de 10 à $50 \mathrm{~Hz}$.

Cette technique de mesure des très faibles vitesses par DQLCE nécessite une détection hétérodyne ou un système de vélocimétrie Doppler $[2,3]$. C'est cette dernière méthode expérimentale qui sera développée dans ce texte.

Dans un précédent article [4], nous avions étudié la mobilité électrophorétique des polyélectrolytes avec la DQLCE en détection hétérodyne. De très nombreuses recherches ont été faites avec ce prin- cipe, en particulier, dans les équipes de B. Ware [57] et E. Uzgiris $[8,9]$ qui ont étudié essentiellement les macromolécules biologiques en fonction des conditions du milieu.

Après avoir rappelé les principes sommaires de la DQLCE et de la vélocimétrie Doppler sous champ électrique (VDCE), nous décrirons les techniques expérimentales en insistant sur la cellule électrophorétique et sur la détermination des plans stationnaires. Ensuite, des exemples d'application seront développés en particulier dans le domaine des colloïdes et des polyélectrolytes linéaires flexibles.

\section{Théorie et principe.}

2.1 DQLCE. - Les principes et la théorie de la DQLCE ont été exposés de nombreuses fois et sont résumés dans le livre de Berne et Pecora [10]. Les équations de base de la fonction d'autocorrélation des fluctuations de concentration $C(q, t)$ et de la densité spectrale $S(q, \omega)$ d'un système de $N$ particules identiques et isotropes peuvent s'écrire :

$$
\begin{aligned}
C(q, t) & \simeq N^{2} \mathrm{e}^{-i \omega_{0} t} \cdot \mathrm{e}^{-i D q^{2} t} \cdot \mathrm{e}^{ \pm i q \mathrm{~V} t} \\
S(q, \omega) & \simeq N^{2} \frac{D q^{2}}{\left[\left(\omega-\omega_{0}\right) \pm \mathbf{q V}\right]^{2}+\left(D q^{2}\right)^{2}}
\end{aligned}
$$

$q$ est le vecteur d'onde $\left(\mathbf{q}=\mathbf{k}_{\mathrm{I}}-\mathbf{k}_{\mathrm{D}}\right.$ avec $k_{\mathrm{I}}$ et $k_{\mathrm{D}}$ qui représentent les vecteurs d'onde de la lumière incidente et diffusée).

$$
q=\frac{4 \pi n}{\lambda_{0}} \sin \frac{\theta}{2}
$$

$n$ est l'indice de réfraction du solvant,

$\lambda_{0}$ est la longueur d'onde incidente dans le vide,

$\theta$ est l'angle de diffusion,

$D$ est le coefficient de diffusion mutuel des particules,

$V$ est la vitesse des macromolécules. 
Si le mouvement se fait perpendiculairement à la direction de la lumière incidente $\mathbf{q} \cdot \mathbf{V}=$ $q V \cos \theta / 2$.

En détection hétérodyne, le spectre obtenu est une Lorentzienne centrée à la fréquence $\left(\omega_{0}-q V \cos \theta / 2\right)$ et de largeur égale à $D q^{2}$ (Fig. 1).

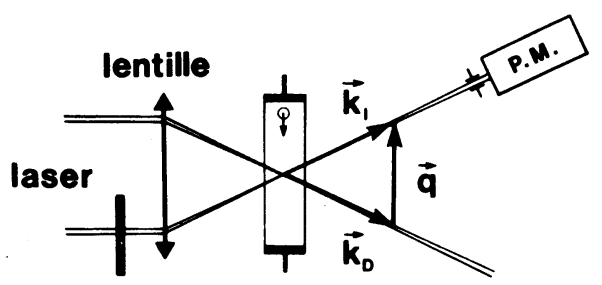

densité neutre
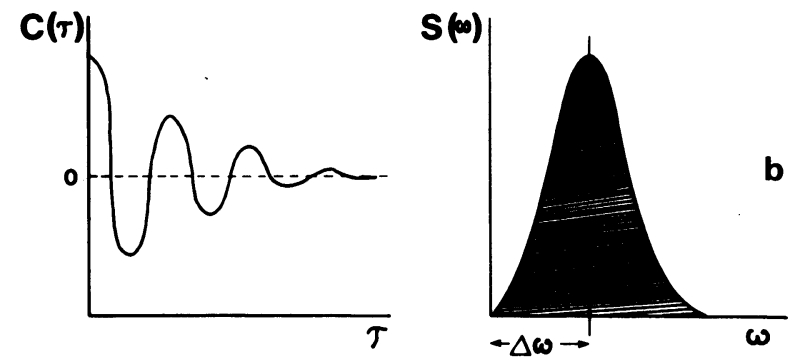

Fig. 1. - a) Représentation schématique de la diffusion quasi élastique sous champ électrique en détection hétérodyne. b) Fonction d'autocorrélation de l'intensité diffusée avec modulation de l'effet Doppler et spectre en fréquence de l'intensité diffusée déplacé par effet Doppler.

[a) Schematic representation of Electrophoretic light scattering in heterodyne detection. b) Autocorrelation function of scattered intensity with Doppler modulation and frequency spectrum of scattered intensity shifted by Doppler effect.]

Ainsi dans une expérience de DQLCE on peut déterminer à la fois les deux paramètres de transport $D$ et $U$. La résolution de la technique est alors :

$$
R=\frac{\Delta \omega}{2 \Gamma}
$$

$\Delta \omega$ est le déplacement en fréquence,

$\Gamma$ est la demi-largeur du spectre diffusé.

Pour une détection à très petits angles, on obtient :

$$
R=\frac{V}{D} \frac{\lambda_{0}}{4 \pi n \theta}=\frac{U E}{D} \frac{\lambda_{0}}{4 \pi n \theta} .
$$

Une résolution convenable $(\sim 5)$ nécessite des particules de taille importante et une détection à de très petits angles.

2.2 VDCE [11, 12]. - La vélocimétrie Doppler sous champ électrique consiste à faire converger au point de mesure de la vitesse électrophorétique, deux faisceaux monochromatiques cohérents. A leur point de croisement, il se forme un système de franges réelles dont l'interfrange est :

$$
i=\frac{\lambda_{0}}{2 \sin \theta / 2}
$$

$\theta$ est l'angle entre les deux faisceaux I et II (Fig. 2).

Si les particules diffusantes traversent l'ensemble des franges avec une vitesse $V$ faisant un angle $\alpha$ avec la bissectrice extérieure de l'angle $\theta$, la lumière diffusée est alors modulée à la fréquence :

$$
\nu=\frac{V \cos \alpha}{i}=\frac{2 V \cos \alpha \sin \theta / 2}{\lambda_{0}} .
$$
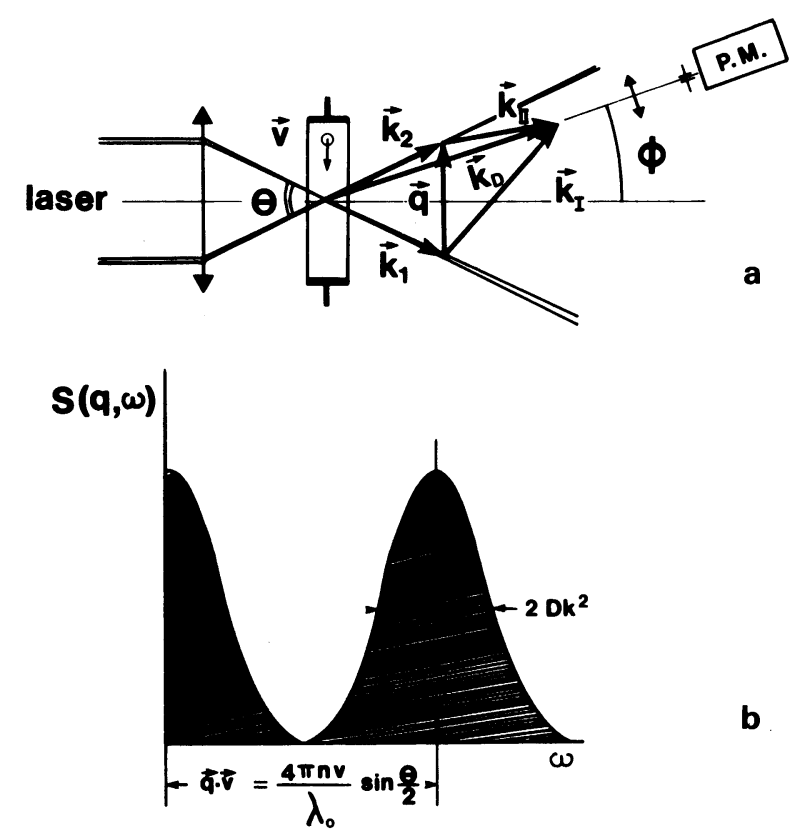

b

Fig. 2. - a) Représentation schématique de la vélocimétrie Doppler sous champ électrique. $\mathbf{k}$ est le vecteur d'onde de diffusion des deux faisceaux incidents $\mathbf{k}=\left|K_{\mathrm{I}}\right|=\left|K_{\mathrm{II}}\right|$. q est le vecteur d'onde entre les deux faisceaux $\left(q=k_{2}-k_{1}\right)$. $q V$ représente le décalage Doppler dû au mouvement des particules. $\Theta$ : angle entre les deux faisceaux. b) Spectres en fréquence de l'intensité diffusée. Cette détection homodyne donne un spectre centré à zéro et un décalage Doppler proportionnel au champ électrique appliqué. Le déplacement Doppler est indépendant de l'angle d'observation, alors que la largeur du spectre diffusé dépend de cet angle.

(a) Schematic representation of the Doppler velocimetry under electric field $\mathbf{k}$ is the scattered wave number of incident beams $\mathbf{k}=\left|K_{\mathrm{I}}\right|=\left|K_{\mathrm{II}}\right| . \mathbf{q}$ is the wave number between the incident and scattered beams, $q=\left(k_{2}-k_{1}\right)$. qV represents the Doppler shift due to the particle motion. $\Theta$ is the scattered angle. b) Frequency spectrum of scattered intensity. Homodyne detection gives a spectrum centred at zero frequency and the Doppler shift is proportional to applied electric field. The Doppler shift is scattered angle independent but the width of scattered spectrum is scattered angle dependent.] 
Une analyse quantitative plus rigoureuse consiste à calculer précisément la fonction d'autocorrélation (FAC) de l'intensité diffusée (qui est la transformée de Fourier, grâce au théorème de Wiener-Khintchine, du spectre de puissance de l'intensité observée). Cette FAC de l'intensité diffusée est reliée simplement à la FAC du champ électrique diffusé. Par un formalisme identique à celui développé par Berne et Pecora [10] à propos de la DQLCE en détection hétérodyne, nous avons pu montrer que dans le cas d'une solution de particules sphériques en concentration suffisamment faible, le spectre de puissance de l'intensité comporte 2 termes :

- une première lorentzienne

$$
\frac{2 D k^{2}}{\omega^{2}+\left(2 D k^{2}\right)^{2}}
$$

centrée en $\omega=0$, de largeur à mi-hauteur $\Delta \omega=$ $2 D k^{2}$ et qui correspond au spectre classique en détection homodyne,

- une seconde lorentzienne

$$
\frac{2 D k^{2}}{(\omega-q \cdot v)^{2}+\left(2 D k^{2}\right)^{2}}
$$

centrée en $\omega=q \cdot v$ et de même largeur que la précédente.

$\mathbf{k}=\left|k_{\mathrm{I}}\right|=\left|k_{\mathrm{II}}\right|$ représente le vecteur d'onde de diffusion des deux faisceaux incidents

$$
\begin{array}{ccc}
\mathbf{k}_{\mathrm{I}}=\mathbf{k}_{\mathrm{D}}-\mathbf{k}_{1} & \text { et } & \mathbf{k}_{\mathrm{II}}=\mathbf{k}_{\mathrm{D}}-\mathbf{k}_{2} \\
\mathbf{q}=\mathbf{k}_{2}-\mathbf{k}_{1} \quad \text { (voir Fig. 2) }
\end{array}
$$

$\mathbf{q} \cdot \mathbf{v}$ représente le décalage Doppler dû au mouvement d'ensemble des macromolécules ; c'est le même que celui observé en détection hétérodyne. Ce décalage en fréquences est indépendant de la direction d'observation et ne dépend que de l'angle entre les deux faisceaux incidents :

$$
\mathbf{q v}=\frac{4 \pi n V}{\lambda} \sin \frac{\theta}{2} \quad \text { pour } \quad \alpha=90^{\circ}
$$

et

$$
\Delta \nu_{(\mathrm{Hz})}=\frac{2 n V}{\lambda_{0}} \sin \frac{\theta}{2}
$$

La largeur du pic déplacé dépend à la fois de l'angle d'observation $\Phi$ et de $\theta$

$$
\begin{aligned}
\Delta \omega_{\left(\mathrm{rd} \mathrm{s}^{-1}\right)} & =4 D k^{2}= \\
& =\frac{D 32 \pi^{2} n^{2}}{\lambda_{0}^{2}}(1-\cos \theta / 2 \cos \phi) .
\end{aligned}
$$

Enfin la résolution spectrale est de la forme :

$$
R=\frac{V}{D} \frac{\lambda_{0}}{8 \pi n} \frac{\sin \theta / 2}{1-\cos \theta / 2 \cos \phi} .
$$

Le rapport signal sur bruit dans cette méthode de franges est excellent pour de faibles concentrations en diffuseurs mais devient médiocre pour de plus fortes concentrations [11].

Cette technique est d'une mise en œuvre expérimentale relativement aisée et plus simple que la détection hétérodyne à faisceaux séparés. Le très grand avantage est la visualisation précise de la zone de mesure au point de croisement des faisceaux. Ceci permet de choisir la plage désirée dans la cellule électrophorétique et ainsi de mesurer précisément les vitesses de déplacement sur les plans stationnaires. Cette spécificité de montage à «franges » n'a pas d'équivalent dans la détection hétérodyne qui souvent donne des spectres de diffusion très dissymétriques présentant des distributions de vitesses électrophorétiques. Enfin, la possibilité de recueillir la diffusion dans n'importe quelle direction est un atout précieux pour certaines études in situ. Tous ces avantages nous ont incité à employer la VDCE pour mesurer les vitesses locales des macromolécules en fonction du champ électrique et pour en déduire ensuite leurs mobilités électrophorétiques.

\section{Appareillage.}

Le schéma de principe de l'installation, dont quelques constituants seront caractérisés ultérieurement, est représenté figure 3 [12]. Le laser, l'optique, la cellule électrophorétique sont montés sur un banc antivibratoire. Le faisceau laser est séparé en deux rayons d'intensité égale par un prisme séparateur Malvern. L'angle entre les deux faisceaux est réglable entre $0^{\circ}$ et $10^{\circ}$. Un angle de $8^{\circ}$ est généralement utilisé dans les études classiques. La bissectrice de l'angle $\theta$ est perpendiculaire aux faces de la cellule électrophorétique. Un objectif $(f=50 \mathrm{~mm})$ placé

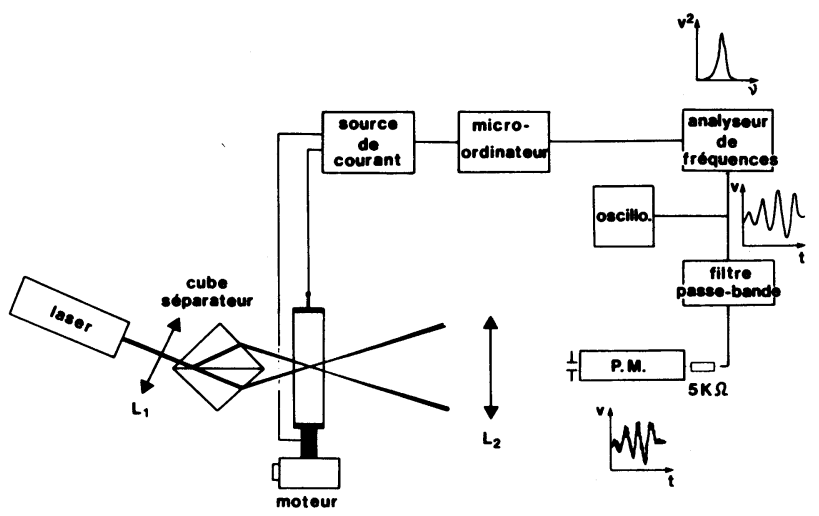

Fig. 3. - Représentation de l'installation de Vélocimétrie Doppler sous champ électrique avec les différentes composantes du montage.

[Representation of the set up of Doppler Velocimetry under electric field with the different components.] 
dans le plan contenant la bissectrice des faisceaux et la normale au plan contenant ces deux rayons reprend la croix d'intersection pour en former l'image sur le diaphragme du photomultiplicateur. Le laser utilisé est un laser Spectra Physics modèle $120 \mathrm{He}-\mathrm{Ne}(5 \mathrm{~mW})$. Le photomultiplicateur est un Hamamatsu R 374 à fenêtre frontale possédant une photocathode type S20.

3.1 Cellule ÉlectrophorétiQue. - Les caractéristiques de la cellule électrophorétique, sont de loin, les plus fondamentales pour avoir des mesures absolues sérieuses de la mobilité électrophorétique. En effet, il faut minimiser, les phénomènes d'électro-osmose, et les perturbations de convection dues au passage du courant électrique. De plus, il est nécessaire d'obtenir une bonne définition du champ électrique au point de mesure et il faut pour cela des surfaces de parois rigoureusement planes et parallèles.

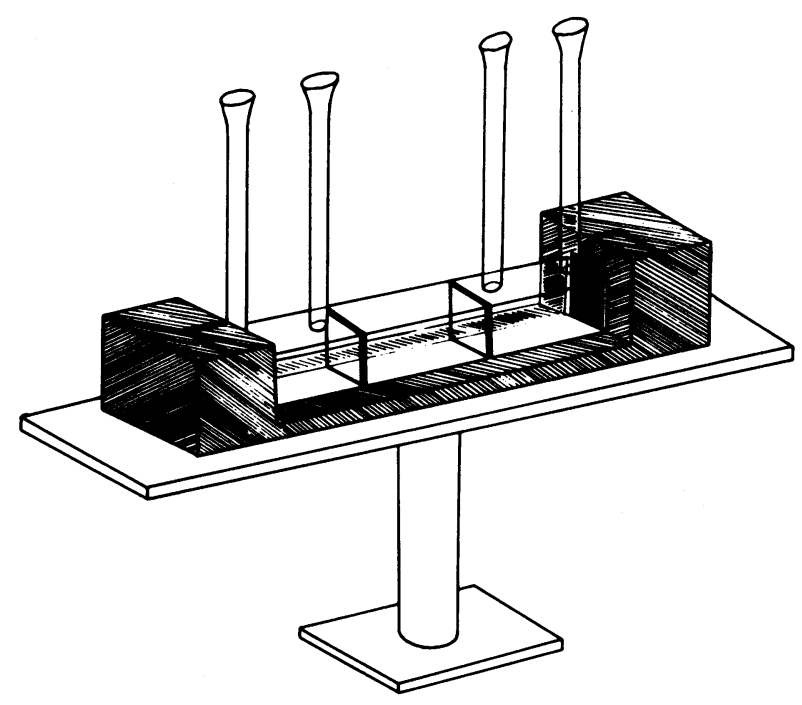

Fig. 4. - Cellule électrophorétique rectangulaire en pyrex ou en quartz (type Helma). Dimensions extérieures : $60 \times 5 \times 3 \mathrm{~mm}^{3}$. Electrode plane en platine. Séparation en trois compartiments par membrane de dialyse.

[Electrophoretic cell having a rectangular shape, made in pyrex or quartz (Helma). External sizes $60 \times 5 \times 3 \mathrm{~mm}^{3}$, platinium Electrodes. The separation is made by a dialysis oriented membrane.]

Nous avons utilisé une cellule simple constituée de trois éléments parallélépipédiques de dimension $(20 \times 5 \times 3) \mathrm{mm}^{3}$ accolés l'un contre l'autre (Fig. 4). Des joints en perbunan ou des membranes de dialyse assurent soit l'étanchéité de l'ensemble soit la séparation des macromolécules vis-à-vis des électrodes de platine qui sont situées aux extrémités.

Avec ce type de cellule, on obtient :

- un bon parallélisme des lignes de champ en utilisant des électrodes planes ;
- une faible élévation de température du fait du grand volume utilisé. Cette cellule peut être utilisée dans un ensemble thermostaté permettant des études en fonction de la température ;

- une bonne définition des plans stationnaires qui permettent d'obtenir des mesures absolues fiables.

Ce dernier point est essentiel dans la définition de la cellule. Les phénomènes d'électro-osmose perturbent considérablement les vitesses des macromolécules. Sous l'application d'un champ électrique, il se crée une atmosphère ionique anisotrope autour du polyion. (Double couche électrique de Gouy). La particule en mouvement entraîne un sillage autour d'elle, constitué d'une couche fine du solvant. De plus, l'absorption des petits ions par les parois de la cellule perturbe le mouvement des polyélectrolytes près de la paroi (mouvement inverse de celui du polyion).

Ce mouvement inverse correspond donc à un écoulement du solvant le long des parois. Ainsi la vitesse de migration des macromolécules sera perturbée soit positivement, soit négativement suivant la position de la mesure. L'évaluation de la vraie vitesse électrophorétique a été possible, théoriquement en résolvant l'équation de Navier-Stokes à l'intérieur de la cellule et expérimentalement par un étalonnage interne. On a pu définir une surface stationnaire où la mobilité perturbatrice (effet des parois) est nulle et des points stationnaires ont été bien localisés.

Komagata [13] a proposé une expression de la vitesse du fluide dans une cellule parallélépipédique dont la hauteur est très grande par rapport à son épaisseur. Nous avons repris le calcul dans le cas de notre cellule, où la hauteur est comparable à son épaisseur. Il est ainsi possible de déduire théoriquement les coordonnées des points stationaires. Les positions calculées, sont dans le plan horizontal median situées à : $1,08 \mathrm{~mm}$ du centre de la cuve et dans le plan vertical médian, ils sont localisés à : $1,94 \mathrm{~mm}$ du centre. Une représentation théorique du profil de vitesse horizontale pour différentes hauteurs sera montrée ultérieurement. Ce type de cellule a été améliorée en utilisant un seul compartiment en quartz pour l'étude des colloïdes qui s'absorbent sur le verre. De plus, des traitements contre l'électro-osmose ont été réalisés pour minimiser les profils de vitesse. Cette cellule électrophorétique est très différente de celle proposée par Ware [14], Schurr [15] et Uzgiris [16].

3.2 GÉNÉRATEUR DE COURANT. - La meilleure manière de calculer le champ électrique est d'appliquer la loi d'Ohm à travers la cellule

$$
E_{\left(\mathrm{V} \mathrm{cm}^{-1}\right)}=\frac{I}{s \chi}
$$


où $I(A)$ est le courant traversant la suspension de conductivité $\chi\left(\mathrm{S} \mathrm{cm}^{-1}\right)$ et $s\left(\mathrm{~cm}^{2}\right)$ représente la section de la cellule au point de mesure.

Pour réduire les effets de polarisation des électrodes, éviter l'électrolyse ou l'échauffement de la solution, il est nécessaire d'utiliser de faibles densités de courant. L'alternance du champ est, de plus, rendue impérative pour supprimer tout gradient de concentration au sein du milieu.

L'utilisation d'une alimentation à courant constant Keithley 220 programmable, permet d'obtenir des courants de sortie allant de $\pm 0,5 \mathrm{pA}$ à $0,1 \mathrm{~A}$ avec un temps de montée inférieur à $3 \mathrm{~ms}$ sous une tension maximum de $100 \mathrm{~V}$.

Dans les expériences de VDCE les courants asservis se situent en général entre $1 \mu \mathrm{A}$ et $1 \mathrm{~mA}$ à $\pm 0,05 \%$. Le milliampère étant pratiquement le courant limite avant l'électrolyse des suspensions aqueuses. Cette alimentation est couplée à un microordinateur Commodore qui synchronise les cycles d'application du champ électrique et l'analyse en fréquences.

Deux types d'application du champ sont utilisés :

(1) Le mode alternatif : le passage du courant est appliqué alternativement dans chaque sens, sans transition, pendant une durée de $2 \mathrm{~s}$. L'analyse en fréquence moyenne alors l'ensemble des modulations sur toute la durée de l'expérience.

(2) Le mode créneau : chaque créneau est appliqué dans un sens selon une durée au moins égale à celle nécessaire pour que l'analyseur effectue une accumulation $(20 \mathrm{~s}$ pour la gamme $0-20 \mathrm{~Hz}, 8 \mathrm{~s}$ pour $0-50 \mathrm{~Hz}$, etc...). Passé ce temps, une séquence de repos est imposée et l'analyse cesse. A l'établissement du créneau de signe opposé, l'analyse est relancée. Un retard dans le déclenchement de l'analyseur peut être éventuellement fixé par l'utilisateur.

Dans ces deux modes, la durée de l'expérience est définie par le nombre de moyennes à effectuer par l'analyseur. Le deuxième mode est utilisé pour des solutions de conductivités élevées, la période de repos limitant l'échauffement (retour à l'équilibre de la solution).

3.3 ANAlyse du Signal de modulation. - Les modulations du courant anodique du photomultiplicateur sont mesurées par la variation de tension de sortie d'une résistance de charge de $5 \times 10^{3} \Omega$. Le signal ainsi collecté passe à travers un filtre actif Krohne Hite 3323 ayant une pente d'atténuation de $24 \mathrm{~dB} /$ oct, utilisé en filtre passe-bande qui élimine les composantes basse fréquence du signal.

Généralement, la fréquence de coupure utilisée sur le canal passe-bas correspond à la fréquence limite de la gamme choisie sur l'analyseur.

A la sortie du filtre passe-bande, la qualité du signal est contrôlée par un oscilloscope. L'analyseur de fréquence utilisé est un Rockland $512 \mathrm{~S}$ 400 canaux à transformée de Fourier rapide, couplé également au micro-ordinateur Commodore.

\section{Test et mode opératoire.}

4.1 DÉTERMINATION DE LA CONSTANTE DE PROPORTIONNALITÉ ENTRE $\Delta \nu$ ET $V$. — La précision de l'angle entre les deux faisceaux étant difficile à évaluer, nous avons dû étalonner le changement de fréquence Doppler connaissant une vitesse de déplacement. Pour cela, la cellule est montée sur un chariot mobile qui se déplace à vitesse connue constante. Une lecture du déplacement en fréquence permet de déduire la constante $\beta$ entre $\Delta \nu$ et $V$. Avec le montage décrit ci-dessus on obtient $\beta=$ $4,60 \mu \mathrm{m} \mathrm{s}^{-1} \mathrm{~Hz}^{-1}$ ce qui correspond bien à un angle de $8^{\circ}$ entre les faisceaux.

Le principal intérêt d'un déplacement simultané de la cellule de mesure est qu'il est possible de déduire le signe de la charge des macromolécules. En effet suivant la polarité des électrodes le sens du déplacement Doppler résultant permettra de connaître le signe de la charge. Enfin, la possibilité de déplacer artificiellement le spectre diffusé peut, dans d'autres cas, améliorer la précision des mesures lorsque les vitesses de migration des molécules sont trop faibles. On évite ainsi la superposition du pic homodyne central et du spectre faiblement déplacé.

\subsection{DÉTERMINATION EXPÉRIMENTALE DES PLANS} STATIONNAIRES. - Nous avons utilisé le déplacement de billes de polystyrène de latex de $1,09 \mu \mathrm{m}$ de diamètre. L'angle d'observation $\phi$ est $20^{\circ}$ dans l'air $\left(15^{\circ}\right.$ dans la solution suite à la réfraction) et ainsi la largeur du pic déplacé est de $3 \mathrm{~Hz}$. Les profils de vitesses des particules sont présentés sur les figures $5 \mathrm{a}$ et $\mathrm{b}$, (a) correspond aux mesures dans le plan horizontal médian et (b) aux mesures dans le plan vertical médian. Les courbes théoriques ont été calculées à partir des équations de Navier-Stokes. Un très bon accord est obtenu entre les profils de vitesse expérimentaux et calculés. Enfin un dernier test a été fait sur un échantillon de dioxyde de titane sous forme de solution colloïdale dont on connaît la valeur du potentiel $\left(45 \mathrm{mV}\right.$ à $\left.21^{\circ} \mathrm{C}\right)$. La détermination des points de la cellule où la valeur de la mobilité correspond à ce potentiel se situe approximativement à $2 \mathrm{~mm}$ du centre dans le plan vertical et à $1 \mathrm{~mm}$ dans le plan horizontal.

Ainsi les expériences seront faites en général dans le plan vertical médian à $2 \mathrm{~mm}$ du centre où la distribution de vitesse est moins étalée dans cette position. Les dimensions caractéristiques du volume de mesure sont de l'ordre de quelques microns carrés et de ce fait la distribution des vitesses est bien définie. 


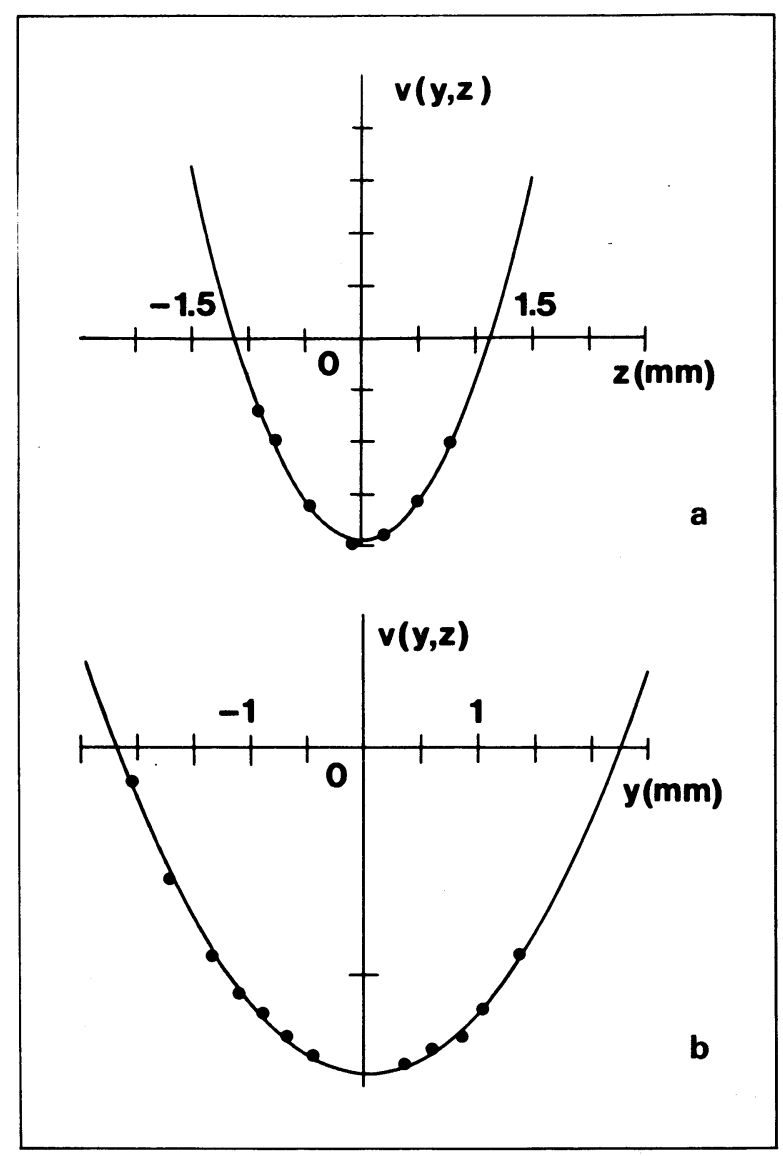

Fig. 5. - a) Profils théorique et expérimental de vitesse dans le plan horizontal médian de la cellule pour la détermination des points stationnaires. b) Profils théorique et expérimental de vitesse dans le plan vertical médian de la cellule pour déterminer les points stationnaires.

(a) Theoretical and experimental profils of velocity in the horizontal plane into the cell for determining the stationaries points. b) Theoretical and experimental profils of velocity in the vertical plane into the cell for determining the stationaries points.]

4.3 LARGEURS DU PIC CENTRAL ET DU PIC DÉPLACÉ. - D'après la théorie exposée précédemment, la largeur du pic central ou déplacé dépend à la fois de l'angle $\theta$ entre les faisceaux et de l'angle d'observation $\phi$

$$
\begin{aligned}
& \Delta \nu_{(\mathrm{Hz})}=\frac{4 D k^{2}}{2 \pi}= \\
& =\frac{16 \pi n^{2} D}{\lambda_{0}^{2}}(1-\cos \theta / 2 \cos \phi) .
\end{aligned}
$$

Nous avons fait varier $\phi$ dans l'air de $15^{\circ}$ à $40^{\circ}$ et mesuré la largeur du pic central pour une solution collö̈dale de sphères de silice de $165 \AA$ (Ludox HS 40, de Dupont de Nemours). On observe bien une évolution de la largeur du spectre en $k^{2}$ et la valeur mesurée correspond approximativement à la valeur calculée. Dans cette technique de VDCE, la largeur du pic déplacé dépend de l'angle d'observation, tandis que son déplacement est seulement sensible à l'angle de croisement des faisceaux.

\subsection{Mode opÉrATOIRE.}

4.4.1 Préparation de la cellule. - Un traitement de la cellule d'électrophorèse est effectué avant son utilisation. Cette préparation a pour simple but d'abaisser la tension de surface paroi/liquide afin de faciliter les opérations de nettoyage et de rinçage. Une réduction légère du profil des vitesses de l'électro-osmose est remarquée, mais ne l'élimine pas. Ce traitement est réalisé à l'aide d'un organosilane Prosil 28 PCR Research Chemicals Inc. conformément aux instructions du fabricant.

La conductivité des suspensions est déterminée à l'aide d'un conductimètre à lecture numérique Tacussel CD 810 possédant une précision électrique meilleure que $\pm 0,25 \%$.

4.4.2 Détermination des mobilités. - La cellule est montée sur son banc optique et, à l'aide de microdéplacements, l'intersection des faisceaux laser est placée sur le plan stationnaire inférieur vertical à la cote $-2 \mathrm{~mm}$ comme indiqué précédemment. L'utilisation d'une loupe binoculaire est nécessaire pour parfaire les réglages de positionnement.

Eventuellement, une vérification de la constante $\beta$ est effectuée par une translation latérale du système en utilisant le moteur.

L'introduction des paramètres expérimentaux s'opère à partir du clavier du micro-ordinateur :

- conductivité $\chi$ de la suspension,

- valeur du courant $I$, et son temps d'application,

- le retard éventuel du déclenchement de l'analyse,

- la constante d'appareillage $\beta$,

- le mode d'application des créneaux.

L'analyse s'exécute ensuite selon le nombre d'accumulations imposé à l'analyseur Rockland. Finalement, après transfert des données de l'analyseur au calculateur, on affiche sur l'écran la valeur du champ élecrique appliqué $(E)$, le glissement de fréquence $(\Delta \nu)$, la vitesse $(v)$ et la mobilité moyenne $(\dot{U})$ ainsi que l'histogramme des mobilités. Une lecture directe des fréquences de déplacement peut être obtenue par l'activation d'un curseur sur l'analyseur ; fréquence, vitesse et mobilité s'affichent alors instantanément sur le calculateur. Pour chaque suspension, plusieurs valeurs de champ sont appliquées. La pente de la droite $\Delta \nu=f(E)$ détermine ainsi la mobilité.

\section{Applications physico-chimiques et discussion.}

5.1 MESURE DE LA MOBILITÉ ÉLECTROPHORÉTIQUE ET DE LA CHARGE EFFECTIVE DE SOLUTIONS COLLOÏDALES [17, 18]. — La mobilité électrophorétique 
$U$ est reliée à la charge effective $Z_{\text {eff }}$ de la macromolécule par l'intermédiaire du coefficient de friction $f$

$$
U=\frac{Z_{\text {eff }} \boldsymbol{e}}{f} \phi(\kappa)
$$

$Z_{\text {eff }}$ est exprimée en unités de charge de l'électron.

$\phi(\kappa)$ est une fonction de la force ionique ou de la longueur de Debye $\left(\kappa^{-1}\right)$

$$
\kappa^{2}=4 \pi L_{B} \sum C_{\mathrm{i}} Z_{\mathrm{i}}^{2} .
$$

$L_{\mathrm{B}} \quad$ est la longueur de Bjerrum $(7,14 \AA$ dans l'eau à $298 \mathrm{~K}$ ),

$Z_{\mathrm{i}} \quad$ est la charge des ions $\mathrm{i}$,

$C_{\mathrm{i}} \quad$ est la concentration en nombre par unités de volume.

Pour des formes globulaires de rayon $R$, la mobilité peut être exprimée par la loi de Henry

$$
U=\frac{Z_{\mathrm{eff}} e}{f} \frac{X(\kappa R)}{1+\kappa R}
$$

avec

$$
X(\kappa R)=1 \quad \text { si } \quad \kappa R \ll 1
$$

et

$$
X(\kappa R)=3 / 2 \quad \text { si } \quad \kappa R \gg 1 .
$$

La charge effective $Z_{\text {eff }}$ peut être déduite de l'équation (16), mais en première approximation il est intéressant d'éliminer le facteur de friction $f$ en mesurant le coefficient de diffusion translationnel

$$
D=\frac{k T}{f} \text {. }
$$

On en déduit la relation de Nernst-Einstein :

$$
Z=\frac{U}{D} \frac{k T}{e} .
$$

Cette relation est strictement valable seulement dans la limite de la dilution infinie c'est-à-dire en absence des interactions entre les espèces ioniques. On obtient alors une valeur de la charge structurale de la macromolécule.

A concentration finie, on mesure une charge effective qui tient compte des conditions de non idéalité de la solution. Cette notion de charge effective, bien qu'imprécise, rend bien compte du phénomène de condensation des contre-ions sur les polyélectrolytes.

Le potentiel peut se déduire de la valeur de la mobilité électrophorétique en utilisant l'équation de Smoluchowski :

$$
\zeta=\frac{\eta U}{\varepsilon}
$$

$\varepsilon$ est la constante diélectrique de l'eau, $\eta$ est la viscosité du fluide.
D'une manière pratique à $t=25^{\circ} \mathrm{C}$ et dans l'eau :

$$
\zeta_{(\mathrm{mV})}=12,8 \times U\left(\mu \mathrm{m} \mathrm{s}^{-1} / \mathrm{V} \mathrm{cm}^{-1}\right) .
$$

- Etude d'un sol d'hydroxyde d'aluminium $\mathrm{Al}(\mathrm{OH})_{3}$. - La décomposition d'un oxalate ou d'un alcoolate d'aluminium peptisé à $\mathrm{pH} \sim 3$ donne une solution colloïdale homogène. La mesure du coefficient de diffusion d'une telle solution à $0,6 \mathrm{~g} / \mathrm{l}$ est de $1,2 \times 10^{-7} \pm 0,1 \mathrm{~cm}^{2} \mathrm{~s}$ soit un rayon hydrodynamique moyen de l'ordre de 200-220 $\AA$.

La valeur de la mobilité électrophorétique obtenue sur les plans stationnaires de la cellule est de $4 \pm 0,2 \times 10^{-4} \mathrm{~cm}^{2} \mathrm{~s}^{-1} \mathrm{~V}^{-1}$. Le potentiel $\zeta$ est donc de l'ordre de $50 \mathrm{mV}$ et la charge effective des colloïdes est, d'après la formule (20), d'environ 100 unités de charge $\left(Z_{\text {eff }} 100\right)$. Cette valeur expérimentale de la charge apparente représente l'ionisation moyenne des particules en suspension. L'application de la relation (20) nécessitant $\kappa R<1$, ne peut se faire qu'à concentration très faible et sans doute très inférieure à $0,6 \mathrm{~g} / \mathrm{l}$. Des expériences, faites à forte dilution, ne montrent pas d'évolution notable des valeurs de $U$ et de $D$, de sorte que la charge apparente déduite $(Z \simeq 100)$ est une caractéristique physique de la solution.

Le signe de la charge a été déduit du mouvement simultané des diffuseurs et du moteur de référence. On observe une charge positive pour les colloïdes à $\mathrm{pH} \simeq 3$.

Nous avons étudié la modification du potentiel avec le $\mathrm{pH}$ pour suivre la gélification de la solution. A $\mathrm{pH} \simeq 5$ pour une concentration de $60 \mathrm{~g} / 1$ on obtient un gel réversible transparent. La mesure de $U$ en fonction du $\mathrm{pH}$ ou de la salinité peut caractériser la transition sol-gel en définissant plus correctement les potentiels de surface.

- Etude de sols de $\mathrm{TiO}_{2}$. - Une étude similaire à la précédente a été faite sur un sol d'oxyde de titane à deux valeurs du $\mathrm{pH}$. Les résultats obtenus sont les suivants :

$$
\begin{array}{cl}
\text { à } \quad \mathrm{pH}=2,5 & \begin{array}{l}
\text { Diamètre des colloïdes } 430 \AA \\
\text { Mobilité électrophorétique : } \\
3 \times 10^{-4} \mathrm{~cm}^{2} / \mathrm{s} / \mathrm{V}
\end{array} \\
& \text { Charge }:+80 \\
& \text { Diamètre des colloïdes } 500 \AA \\
& \text { Mobilité électrophorétique : } \\
7 \times 10^{-4} \mathrm{~cm}^{2} / \mathrm{s} / \mathrm{V} \\
\text { Charge }:-200 .
\end{array}
$$

Il est donc possible de suivre l'évolution de la mobilité électrophorétique en fonction $\mathrm{du} \mathrm{pH}$, de déduire le $\mathrm{pH}$ isoélectrique et ainsi de mieux maîtriser la stabilité des solutions colloïdales.

5.2 MOBILITÉ ÉLECTROPHORÉTIQUE DES MACROMOLÉCULES LINÉAIRES ET FLEXIBLES. - La théorie de la mobilité électrophorétique de chaînes linéaires 
chargées a été proposée par Henry en 1931 [20] et reprise récemment par Manning en 1975 [21] et 1982 [22]. La théorie de Manning est applicable en solution sans sel [21] ou en excès de sel [22]. Les deux effets d'électrophorèse et de relaxation (ou champ d'asymétrie) y sont calculés. Le premier conduit, pour un contre-ion monovalent à l'expression suivante :

$$
300\left|U^{*}\right|\left(\mathrm{cm}^{2} \mathrm{~s}^{-1} \mathrm{~V}^{-1}\right)=\frac{1}{z_{\mathrm{c}}} \frac{\varepsilon k T}{3 \pi \eta e}|\ln (\kappa a)|
$$

$\kappa$ est l'inverse de la longueur de Debye. $Z_{\mathrm{c}}$ est la valence du contre-ion, $a$ est la distance entre sites chargés du polyion. Cette relation se réduit à l'expression suivante dans l'eau et à $20^{\circ} \mathrm{C}$ :

$$
\left|U^{*}\right|=2,38 \times 10^{-4}|\ln \kappa a| .
$$

La relation complète en tenant compte des deux corrections dues à la force ionique peut alors se mettre sous la forme :

$$
U=U^{*} \frac{\alpha}{\beta} .
$$

Pour une solution sans électrolyte à deux composants (polyion et contre-ion)

$$
\frac{\alpha}{\beta}=\frac{0,866}{1+43,3\left(\frac{300 U^{*}}{\lambda_{c}^{0}}\right)}
$$

$\lambda_{\mathrm{c}}^{0}$ représente la conductance équivalente du contreion dans l'eau pure.

- Pour une solution de polyélectrolyte avec un excès de sel $(\mathrm{NaCl})$ on écrit alors :

$$
\begin{aligned}
\frac{\alpha}{\beta} & =\frac{1}{1+3,86|\ln \kappa a|\left[\frac{1}{\lambda_{\mathrm{Na}^{+}}^{0}}+\frac{1}{\lambda_{\mathrm{Cl}^{-}}^{0}}\right]} \\
& =\frac{1}{1+0,142|\ln \kappa a|} .
\end{aligned}
$$

D'après ces formules on voit que le paramètre

$$
\frac{\left|U^{*}\right|}{|\ln \kappa a|}
$$

est une constante pour un solvant donné et à une température fixée. Celui-ci vaut $2,38 \times$ $10^{-4} \mathrm{~cm}^{2} \mathrm{~s}^{-1} \mathrm{~V}^{-1}$ en solution aqueuse à $20^{\circ} \mathrm{C}$ avec des contre-ions monovalents.

En tenant compte de l'effet d'asymétrie du champ électrique on obtient une légère évolution du rapport $\frac{\left|U^{*}\right|}{|\ln \kappa a|}$ en fonction de la concentration.

Le formalisme d'Henry pour des faibles valeurs de $K r$ ( $r$ étant le rayon du polyélectrolyte supposé cylindrique) se rapproche de celui de Manning à la différence que Manning considère un polyélectrolyte infiniment long.

La formule simplifiée d'Henry peut s'écrire

$$
300\left|U^{*}\right|\left(\mathrm{cm}^{2} \mathrm{~s}^{-1} \mathrm{~V}^{-1}\right)=\frac{\varepsilon k T}{4 \pi \eta e}|\ln \kappa r|
$$

donc $\frac{\left|U^{*}\right|}{|\ln \kappa r|}$ est une constante et dans ce cas la mobilité dépend uniquement du rayon du polyélectrolyte alors que dans la théorie de Manning $U^{*}$ dépend spécifiquement de la distance entre les charges.

5.2.1 Mobilité électrophorétique du polystyrène sulfonate de sodium (PSSNa) [12/23]. — Le polystyrène sulfonate de sodium provient de Pressure Chemical Co. La masse moléculaire de l'échantillon étudié est de $7,8 \times 10^{5} \mathrm{~g} \cdot \mathrm{mol}^{-1}$ et la longueur de la chaîne est de l'ordre de $1 \mu \mathrm{m}$. La distance entre les charges est de $2,5 \AA$. Il est dissous dans de l'eau désionisée $(18 \mathrm{M} \Omega \mathrm{cm})$ et introduit dans la cellule électrophorétique.

- Evolution de $U$ avec la concentration en polyélectrolyte $(C)$ en absence de sel. - La figure 6a montre la variation de $U$ en fonction de $C$. On note la présence d'un «cross-over » à $2,5 \times 10^{-2} \mathrm{~g} / 1$. Deux comportements ont été déduits par rapport à

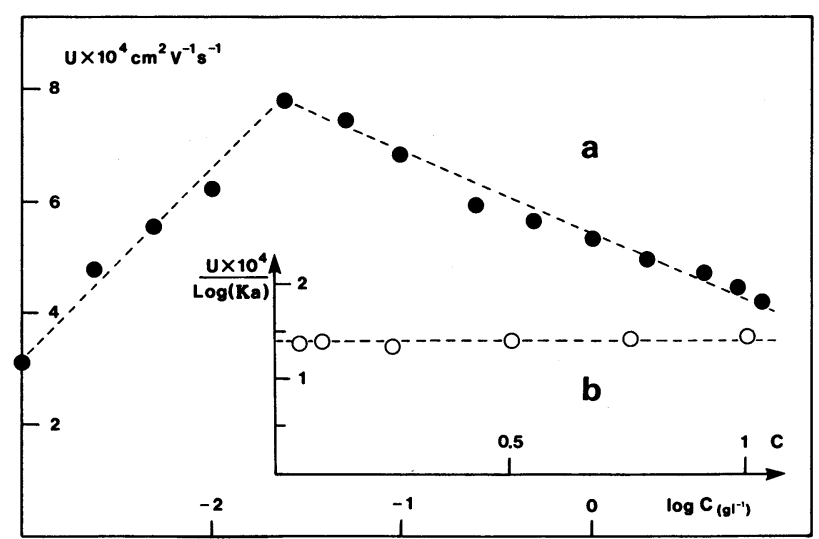

Fig. 6. - a) Evolution de la mobilité électrophorétique $\mathrm{du}$ polystyrène sulfonate de sodium en fonction de la concentration (solution sans sel). Les valeurs ont été obtenues sur les plans stationnaires de la cellule $C^{*} \simeq$ $2,5 \times 10^{-2} \mathrm{~g} /$. b) Evolution du paramètre $\frac{U}{|\ln \kappa a|}$ en fonction de $C$ en accord avec la théorie de Manning tenant compte de la correction de champ asymétrique.

[a) Evolution of the electrophoretic mobility in sodium polytyrene sulfonate solution as a function of polyelectrolyte concentration (free salt solution). The values have been obtained on the stationaries plans of the cell $C^{*}=2.5 \times 10^{-2} \mathrm{~g} / \mathrm{l}$. b) Evolution of $\frac{U}{|\ln \kappa a|}$ in agreement with the Manning's theory which takes into accounts the asymetric field correction.] 
cette concentration qui correspond approximativement à la concentration critique de recouvrement $C^{*}$.

$C<C^{*}: U$ croît avec $C$ en $C^{1 / 3}$

$C>C^{*}: U$ décroît avec $C$ suivant la théorie de Manning. Dans le cas d'une solution sans sel :

$$
\kappa^{2}=4 \pi a C .
$$

Pour des valeurs finies de $C$, on peut donc calculer $\kappa$ et la valeur de $\frac{\left|U^{*}\right|}{|\ln \kappa a|}$.

La figure $6 \mathrm{~b}$ représente l'évolution de ce paramètre qui est indépendante de $C$ et la valeur est de l'ordre de 1,70 en accord avec le formalisme de Manning prenant en compte la correction du champ asymétrique. Cette expérience confirme la relation entre la mobilité et la distance inter-charges. Ainsi le formalisme d'Henry ne permet pas d'ajuster correctement les valeurs expérimentales car le diamètre du polyélectrolyte doit être au moins de l'ordre de 8 à $10 \AA$. Des études identiques faites avec d'autres masses moléculaires ont montré que la mobilité ne dépend pas du degré de polymérisation [24], mais, par contre, elle est très sensible à la distance entre les charges [11].

- Evolution de $U$ en fonction de sel ajouté $\left(\overline{\left.C_{\mathrm{s}}\right) .}-\right.$ Nous avons étudié la variation de $U$ en fonction de la concentration en sel ajouté $\mathrm{NaCl}$ pour deux concentrations en PSSNa (Figs. 7a et 7b). Les valeurs expérimentales de $U$ décroissent avec $C_{\mathrm{s}}$ et suivent qualitativement la théorie de Manning (traits pleins). Tous les résultats expérimentaux obtenus sur les polyélectrolytes linéaires flexibles se situent généralement entre les valeurs théoriques $U^{*}$ et $U$. De ce fait, la différence entre les valeurs théoriques et expérimentales peut être attribuée : soit à une évaluation théorique trop importante du ralentissement du polyion par l'effet de relaxation, soit à une distance entre charges supposée trop grande par rapport à sa valeur réelle.

- Evolution de la mobilité à $K$ constant. - Le paramètre de Manning $=\frac{L_{\mathrm{B}}}{a}=2,8$ permet de prévoir une fraction condensée de $64 \%$ d'ions $\mathrm{Na}^{+}$ [25]. Dans ce but, nous avons effectué deux séries de mesures à $\kappa$ constant en faisant varier à la fois les concentrations du polyions $(C)$ et du sel $\left(C_{\mathrm{s}}\right)$ de manière à maintenir $\kappa$ constant suivant la relation :

$$
\kappa^{2}=4 \pi a C\left[1+\frac{2 C_{\mathrm{s}}}{C}\left(\frac{a}{L_{\mathrm{B}}}\right)\right] .
$$

Les points expérimentaux $\square$ mesurés en considérant la condensation de Manning sont indépendants de $\kappa$ quelle que soit la concentration en polyions. Cette évolution est en parfait accord avec la théorie.
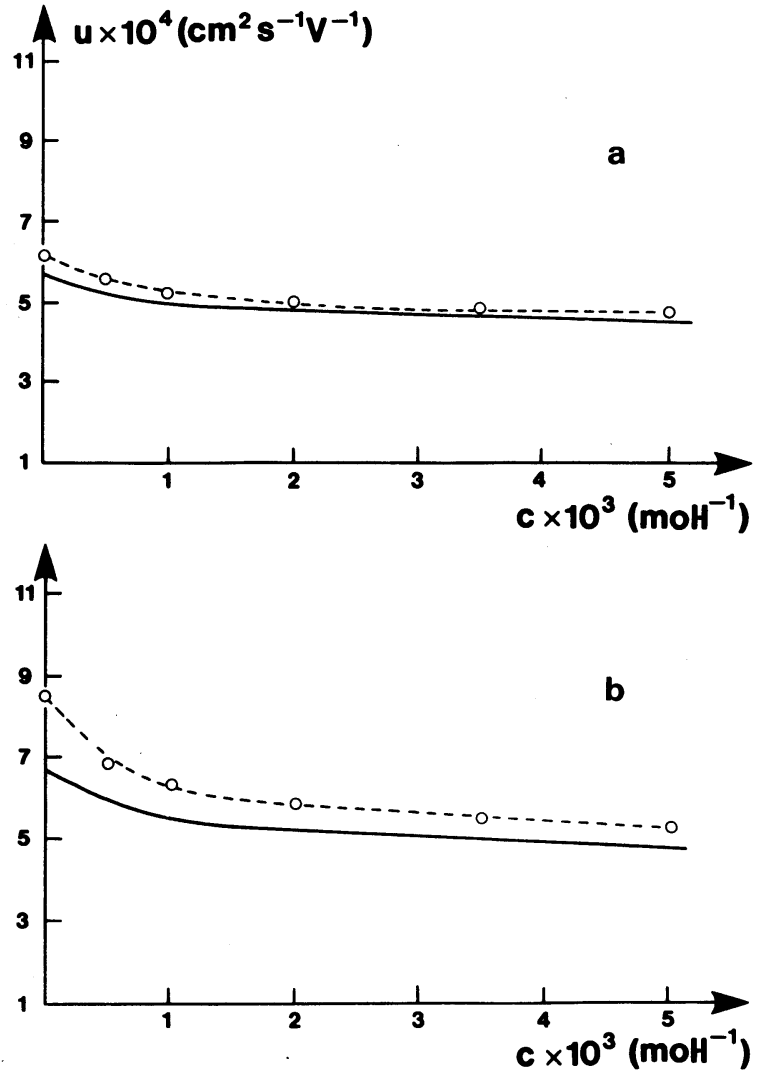

Fig. 7. - Evolution de la mobilité électrophorétique de PSSNa en fonction de la concentration du sel ajouté $\left(C_{\mathrm{s}}\right)$. a) Concentration en PSSNa $C_{\mathrm{p}}=4 \times 10^{-2} \mathrm{~g} /$. b) Concentration en PSSNa $C_{\mathrm{p}}=0,5 \mathrm{~g} / \mathrm{l}$. $\mathrm{O}:$ Points expérimentaux. La ligne continue représente l'évolution théorique de Manning.

[Variation of the electrophoretic mobility of NaPSS as a function of added salt $\left(C_{\mathrm{s}}\right)$. a) Polyelectrolyte concentration : $C_{\mathrm{p}}=4 \times 10^{-2} \mathrm{~g} / \mathrm{l}$. b) Polyelectrolyte concentration : $C_{\mathrm{p}}=0.5 \mathrm{~g} / \mathrm{l}$.]

Au contraire, si on considère une ionisation totale du PSSNa sans tenir compte de la condensation, les points expérimentaux $\bullet$ évoluent avec la concentration en polyélectrolytes. Bien que $\kappa$ soit constant, la variation de $U$ avec $C$ met en évidence l'importance de la notion de condensation des contre-ions dans les solutions de polyélectrolytes [25].

Des illustrations identiques ont été faites sur des polysaccharides $[26,27]$ (sulfate de chondroïtine, héparine, $\kappa$-Carragenane sur des protéines [28]. Généralement, la mobilité électrophorétique a permis de bien maîtriser la notion de charge effective, de mieux définir le phénomène de condensation et de compétition entre des contre-ions de valences différentes [18].

\section{Conclusion.}

La vélocimétrie Doppler sous champ électrique permet d'étudier les propriétés de transport des 


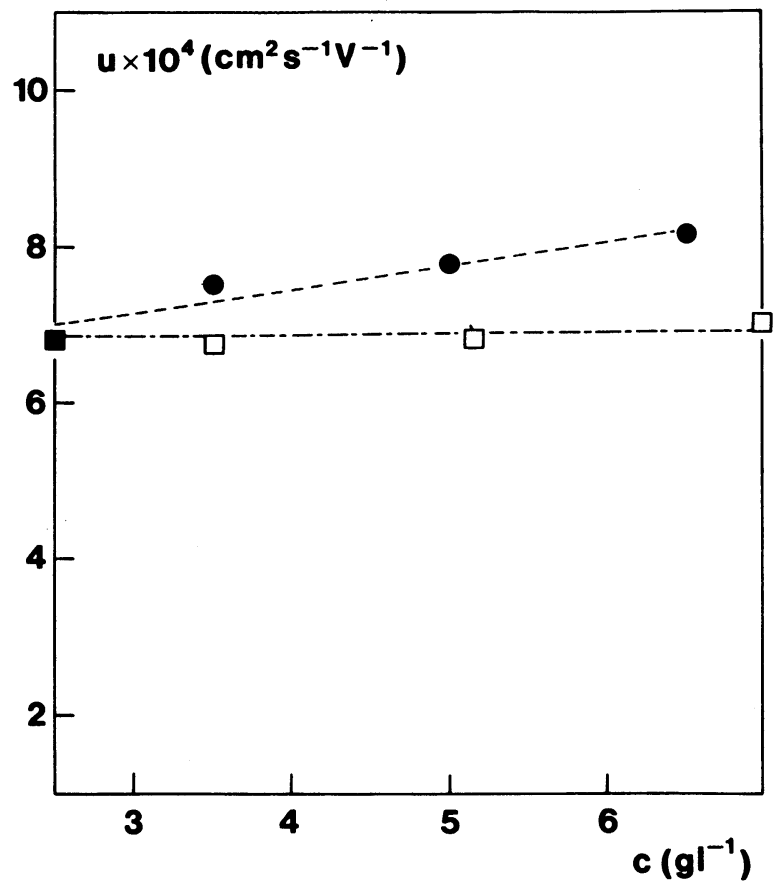

Fig. 8. - Evolution de la mobilité électrophorétique à $\kappa$ constant en fonction de la concentration en PSSNa $(C)$. $\square$ Points expérimentaux mesurés en considérant la condensation des contre-ions suivant la théorie de Manning. - Points expérimentaux mesurés en considérant une ionisation totale du polyion.

[Evolution of the electrophoretic mobility of NaPSS at constant $\kappa$ as a function of polyelectrolyte concentration $\left(C_{\mathrm{p}}\right)$. $\square$ Experimental data with the Manning Condensation of counter ions. - Experimental data without the Manning Condensation of counter-ions.]

macromolécules chargées et de déduire la charge effective des polyions. Nous avons présenté une technique basée sur le principe de la modulation de franges par le mouvement directif des diffuseurs. Celui-ci est détecté et analysé en fréquence en fonction du champ électrique appliqué. L'importance de la géométrie de la cellule électrophorétique et de la définition des plans stationnaires a été mise en évidence. Un avantage tout à fait fondamental de ce montage est qu'il permet des mesures précises sur les points stationnaires - des profils de vitesse. De ce fait, les formes des spectres diffusés sont relativement bien définies et symétriques et les valeurs de la mobilité électrophérique sont plus précises.

La technique de « modulation de franges » semble supérieure à la détection hétérodyne à faisceaux séparés par une meilleure définition du volume diffusant.

Des applications physico-chimiques ont illustrées cette méthode d'étude des propriétés électrocinétiques des solutions colloïdales. La détermination du potentiel $\zeta$, son évolution avec les conditions du milieu ( $\mathrm{pH}$, force ionique, concentration) sont des paramètres importants pour suivre les formations de sols, les transitions sol-gel. Des exemples ont été présentés sur les sols d'hydroxyde d'aluminium et d'oxyde de titane.

Enfin l'hydrodynamique des polyélectrolytes linéaires flexibles peut être appréhendée par cette technique. En effet, l'évolution de la mobilité électrophorétique avec la concentration de polyion est directement corrélée aux interactions hydrodynamiques $(H)$ [29]. On peut alors écrire les trois relations fondamentales dans les techniques de diffusion sur des macromolécules globulaires chargées :

$$
\begin{array}{ll}
\text { - Mobilité } & : U=U_{0}(1+H) \\
\text { - Diffusion } & : D=D_{0}(1+H) / S \\
\text { - Intensité } & : I=P \times S
\end{array}
$$

$S$ et $P$ étant les facteurs de forme et de structure des particules diffusantes.

Ainsi la mobilité électrophorétique associée avec les mesures des coefficients de diffusion et de l'intensité diffusée peut donner une bonne approche des propriétés dynamiques et structurales des polyélectrolytes [23].

L'illustration de ces propriétés a été faite sur le polystyrène sulfonate de sodium. L'évolution de la mobilité avec la concentration met en évidence un « accident » à $C^{*}$. Pour $C<C^{*}$ un ordre local est supposé présent dans la solution et la mobilité croît en $C^{1 / 3}$ jusqu'à la pseudofusion des domaines ordonnés [30]. Pour $C>C^{*}$ l'évolution de la mobilité suit la théorie de Manning c'est-à-dire qu'elle dépend directement de $\kappa$ et de $a$ (distance entre charges). Enfin l'évolution expérimentale de la mobilité électrophorétique en fonction de la concentration en sel ajouté $\left(C_{\mathrm{s}}\right)$ est également en accord avec le formalisme de la condensation des contre-ions.

Pour terminer, nous pouvons ajouter que la mesure de la mobilité électrophorétique est un atout très précieux pour suivre, les phénomènes d'agrégations, les interactions polymères-colloïdes, la dynamique de sondes chargées dans des sols transparents qui évoluent vers la gélification [31]. 


\section{Bibliographie}

[1] Ware, B. R., Flygare, W. H., Chem. Phys. Lett. 12 (1971) 81.

[2] Durst, F., Helling, A., Whitelaw, J. M., Principles and practice of Laser Doppler Anemometry (Ac. press) 1976.

[3] WatrasiewiCs, M. T., Laser Doppler Measurements (Butterworths) 1975.

[4] Drifford, M., Menez, R., Tivant, P., Nectoux, P., Dalbiez, J. P., Rev. Phys. Appl. 16 (1981) 19.

[5] Ware, B., Adv. Colloid Interface Sci. 4 (1974) 1.

[6] SMITH, B. A., WARE, B. R., in Contemporary Topics in Analytical and Chemical Chemistry Vol. 2 edited by D. M. Hercules et al. (Plenum, New York) 1978, p. 29.

[7] WARE, B. R., in « the Application of laser light scattering to the study of biological motion " edited by J. C. Earnshaw et al. Nato adv. Study Inst. Ser. (Plenum, NY) 1983.

[8] Uzgiris, E. E. et KaPlan, J. H., J. Colloid Interface Sci. 55 (1976) 148.

[9] UzGIRIs, E. E., Adv. Colloid Interface Sci. 14 (1981) 75.

[10] Berne, B. J. and Pecora, R., Dynamic light scattering (Wiley, NY) 1976.

[11] Drain, L. E., The Laser Doppler Technique (Wiley, NY) 1980

[12] Taвti, K., Thèse $3^{\mathrm{e}}$ cycle Paris VI, 1983.

[13] Komagata, S., Res. Electrotechn. Lab. Jpn 348 (1933) 5.

[14] Hass, D. D. and Ware, B. R., Anal. Biochem. 74 (1976) 175.
[15] Wilcoxon, J. P. and Schurr, J. M., J. Chem. Phys. 78 (1983) 3354

[16] Uzgiris, E. E., Rev. Sci. Instrum. 45 (1974) 74

[17] Magdalenat, H., Turo, P., Tivant, P., Chemla, M., Menez, R., Drifford, M., J. Chem. Educ. 55 (1978) 12.

[18] Tivant, P., Turq, P., Drifford, M., MagdeleNAT, H., MENEZ, R., Biopolymers 22 (1983) 643.

[19] Hunter, R. J., in Zeta Potential in Colloid Science, Principes and applications (Ac. Press, NY) 1981.

[20] Henry, D. C., Proc. Roy. Soc. London A 133 (1931) 106.

[21] Manning, G., J. Phys. Chem. 79 (1975) 262.

[22] Manning, G., J. Phys. Chem. 85 (1981) 1506.

[23] Drifford, M., Dalbiez, J. P., J. Chim. Phys. 82 (1985) 571.

[24] Meullenet, I., Schmitt, A., Drifford, M., J. Phys. Chem. 83 (1979) 1924.

[25] Manning, G., J. Chem. Phys. 11 (1969) 924.

[26] DRIFFord, M., TIVANT, P., BENCHEIKH-LaRbi, TABTi, K., Rochas, C., Rinaudo, M., J. Phys. Chem. 88 (1984) 1414.

[27] Tivant, P., Thèse d'Etat Paris (1985).

[28] Hantz, E., CaO, A., Talllandier, E., Tivant, P., DRIFFORD, M., DEFER, N., KRUH, J., Intern. J. Biol. Macromol. 5 (1983) 130.

[29] Walrand, S., Belloni, L., Drifford, M., J. Physique 47 (1986) 1565.

[30] Drifford, M., Dalbiez, J. P., J. Phys. Chem. 88 (1984) 5368.

[31] Gauthier-Manuel, Thèse d'Etat Paris (1986). 